\title{
Synuclein in red blood cells: a potential biomarker for multiple system atrophy, and other updates on recent autonomic research
}

\author{
Mitchell G. Miglis ${ }^{1} \cdot$ Srikanth Muppidi ${ }^{1}$
}

Received: 3 March 2020 / Accepted: 4 March 2020 / Published online: 18 March 2020

(c) Springer-Verlag GmbH Germany, part of Springer Nature 2020

\section{Synuclein in red blood cells: a potential biomarker for multiple system atrophy?}

The search for a biomarker in the $\alpha$-synucleinopathies and other neurodegenerative proteinopathies has become the "holy grail" of neurodegenerative research. Identifying a reliable biomarker could not only aid in diagnosis (e.g., differentiating Parkinson disease from multiple system atrophy [MSA]), but also in the prognosis (identifying those with more benign vs. severe forms of disease), and monitoring of disease progression (essential for future clinical trials), thus revolutionizing the field. Among the $\alpha$-synucleinopathies, no disease presents more diagnostic and therapeutic challenges than MSA, the most aggressive subtype of these disorders. Various molecules, including $\alpha$-synuclein ( $\alpha$ Syn), DJ-1, tau, amyloid- $\beta$, neurofilament light chain, dopamine, and neuroinflammatory cytokines, have all been investigated as serum biomarkers for the diagnosis of MSA; however, sensitivity and specificity have been suboptimal due to interference by plasma proteins, contamination by hemolysis, and variations in detection methods. Interestingly, 99\% of $\alpha$ Syn in whole blood is located in the red blood cells (RBCs). Because whole blood avoids the interference problems encountered when measuring cerebrospinal fluid and plasma $\alpha$ Syn, it could be an appealing option for the detection of pathological $\alpha$-synuclein. There have now been several studies that have evaluated different forms of RBC-derived $\alpha$ Syn (e.g., total vs. the pathological phosphorylated subtype); however, because MSA is a rare disorder, studies have been limited by small sample sizes.

In a recent publication in the journal Parkinson's Disease [1], Li and colleagues investigated the levels of

Srikanth Muppidi

muppidis@stanford.edu

1 Stanford Neurosciences Health Center, Stanford Medical Center, 213 Quarry Road, 2nd Floor, Palo Alto, CA 94304, USA phosphorylated $\alpha$ Syn in RBCs in a large MSA cohort (107 patients) and 220 age- and sex-matched controls from China. They used an enzyme-linked immunosorbent assay (ELISA) to quantify the levels of phosphorylated $\alpha$ Syn in RBCs. They also analyzed the correlations of phosphorylated $\alpha$ Syn in RBCs with various clinical variables including disease duration, Hoehn \& Yahr (H\&Y) stage, the Unified Multiple System Atrophy Rating Scale (UMSARS), the Montreal Cognitive Assessment, the Rapid Eye Movement Sleep Behavior Disorder Questionnaire-Hong Kong (RBDQ-HK), and the Hamilton Depression and Anxiety Scales. Age at onset and disease duration was $58 \pm 9$ and $2 \pm 2$ years, respectively. The levels of phosphorylated $\alpha$ Syn in RBCs were significantly higher in patients with MSA compared to controls $(14.02 \pm 4.02$ vs. $11.89 \pm 3.57 \mathrm{ng} / \mathrm{mg}$, $p<0.001)$. In a multivariate logistic regression model, the area under the receiving operating curve (ROC) was 0.91 (95\% confidence interval (CI): 0.87-0.94), with a sensitivity of $80.37 \%$ and a specificity of $88.64 \%$ for the diagnosis of MSA. While these are higher sensitivity and specificity values than those of plasma and CSF $\alpha$ Syn analysis, it should be noted that these prior studies evaluated total $\alpha$ Syn, and not the more specific pathological form of phosphorylated $\alpha$ Syn reported in this study. The authors performed a sub-group analysis of 75 patients who were subclassified into the MSA-P or MSA-C phenotypes of the disease, and found that the levels of phosphorylated $\alpha$ Syn in RBCs were significantly higher in MSA-P vs. MSA-C $(13.27 \pm 1.91$ vs. $12.19 \pm 3.04 ; p=0.025$ ). Interestingly, in a multivariate linear regression analysis, the authors reported a negative correlation between the levels of phosphorylated $\alpha$ Syn in RBC levels and RBDQ-HK scores. The reason for this is not clearly hypothesized by the authors; however, one explanation could involve a heterogeneous cohort, along with heterogenous reporting of RBD behaviors by patients. Other limitations of this study include the lack of comparison between phosphorylated $\alpha$ Syn in red blood cells and other diagnostic groups such as Parkinson disease or dementia 
with Lewy bodies. Of the reported 107 patients, only 34 (31.7\%) were classified as probable MSA, and the remainder possible MSA, creating another limitation and one that has plagued many prior studies in MSA: the lack of a carefully phenotyped cohort. Finally, anemia is not infrequent in patients with autonomic failure, including MSA. The total RBC count was a potential cofounder that was not completely accounted for. While further studies are needed to evaluate the utility of phosphorylated $\alpha$ Syn in red blood cells as a biomarker, either alone or in combination with other biomarkers, this study represents an important effort in the continued quest for the holy grail of the $\alpha$-synucleinopathies.

\section{Monitoring the autonomic nervous system through wearables}

Wearable technology has become ubiquitous in the last decade, with increasing applications in healthcare, most notably in the detection of atrial fibrillation [2] and epilepsy [3]. These wearable devices assess changes in body movement, heart rate, or other physiological variables in patients and can identify target events such as atrial fibrillation or seizures, with potentially life-saving consequences. Despite the obvious application in the field of autonomic medicine, wearable technology has thus far failed to find an appropriate market. More recently, researchers have developed detection methods for autonomic dysreflexia in patients with spinal cord injury, which, like atrial fibrillation and epilepsy, represents a potentially fatal autonomic disease.

In a recent article published in the IEEE Journal of Translational Engineering in Health, Suresh and colleagues report the use wearables for the early recognition of autonomic dysreflexia [4]. The authors used a Microsoft band that captures heart rate, skin temperature, and galvanic skin response. They set up a telemetry system with the help of an application on a portable tablet and remote server, with the option to deliver alerts to the caregiver. To set up real-time monitoring without the need for human interpretation, the authors utilized artificial intelligence and machine learning to improve the overall accuracy of their physiological monitoring system. They recruited 11 patients with autonomic dysreflexia with awareness of their symptoms. In the first 7 patients, changes in heart rate, galvanic skin response and skin temperature were measured throughout the day, and patients were asked to provide feedback on a tablet when they experienced symptoms. With this information, the authors developed a model to predict autonomic dysreflexia, which was then validated in the remaining 4 patients. The method was able to detect the onset of autonomic dysreflexia with an accuracy of $94 \%$, with a false-negative rate of $5 \%$. These findings represent an exciting development in wearable technology, and one that might allow patients life-saving earlier detection of autonomic dysreflexia. The limitations of this study include an extremely small sample size, with an average test data set from only 7 patients. Reliable artificial intelligence and machine learning projects typically require thousands of datasets. Nonetheless, we hope that further development of wearables allows for more seamless monitoring of autonomic dysreflexia in spinal cord injury, along with other autonomic disorders.

\section{Finally, an animal model of pure autonomic failure}

Depending on the type and severity of disease, the $\alpha$-synucleinopathies can manifest with both autonomic and motor manifestations, contingent on the degree and location of $\alpha$ Syn deposition in the central and peripheral nervous system. Pure autonomic failure (PAF) presents with autonomic features such as neurogenic orthostatic hypotension before evolving to other phenotypes with motor or cognitive manifestations, with a mean time to phenoconversion of 12 years. These features make PAF an ideal prodromal syndrome for disease-modifying therapies; however, an animal model would be needed to accelerate drug development.

In a recent publication in Nature Communications, Wang et al. [5] studied the effects of injecting $\alpha$ Syn preformed fibrils into stellate and celiac ganglia in transgenic mice overexpressing human $\alpha$ Syn with the A53T mutation $\left(\mathrm{TgM} 3^{ \pm}\right)$. Following the injections, a series of experiments were performed to study autonomic function, including the presence of orthostatic hypotension, constipation, and reduced sweating, replicating the phenotype of patients with PAF. The goal was to assess the spread of $\alpha$ Syn along the peripheral and central autonomic pathways. Remarkably, mice injected with $\alpha$ Syn had many features of autonomic failure, and these features correlated with the spread of $\alpha$ Syn both centrally and peripherally. The mice developed orthostatic hypotension (measured non-invasively through a blood pressure cuff around the tail), constipation (measured as gastrointestinal transit time after mice were given activated carbon labeled food), anosmia (measured by buried a food test) and anhidrosis (measured by both pilocarpine-induced sweat and starch-iodine assay at fore and hind paws). The authors used double immunostaining for various neural structures (neuron, astrocyte, oligodendrocyte) and $\alpha$ Syn to determine transmission of $\alpha$ Syn along the autonomic pathways. They noticed retrograde migration of $\alpha$ Syn to the CNS, specifically autonomic nuclei in the brainstem, as early as 2 months post-injection with increasing $\alpha$ Syn density with time. There was also anterograde migration of $\alpha$ Syn to the peripheral effector organs such as cardiac tissue, blood vessels, gut, and skin. In addition, unilateral injection of $\alpha$ Syn in autonomic ganglia resulted in unilateral spread. 
Overall, this animal model is a remarkable reproduction of PAF. The main limitation of this study is a short murine life span of only 24 weeks, as this might make assessing the benefit of various therapies difficult. In addition, the authors used transgenic mice overexpressing human $\alpha$ Syn, instead of wild-type mice, which might have resulted in an artificially aggressive form of the disease. Despite these limitations, this mouse model of PAF is a remarkable step in the direction of confirming prior hypotheses of retrograde and anterograde $\alpha$ Syn spread as well as correlation with the phenotype of autonomic failure. Development of $\alpha$ Syn therapies that can be tested on animal models of prodromal $\alpha$-synucleinopathy should not be far away.

Funding None.

\section{Compliance with ethical standards}

Conflict of interest The author(s) declare that they have no conflict of interests.

\section{References}

1. Li XY, Yang W, Li X et al (2020) Phosphorylated Alpha-synuclein in red blood cells as a potential diagnostic biomarker for multiple system atrophy: a pilot study. Parkinson's Dis 24:8740419

2. Perez MV, Mahaffey KW, Hedlin H et al (2019) Large-scale assessment of a smartwatch to identify atrial fibrillation. N Engl J Med 381:1909-1917

3. van Westrhenen A, De Cooman T, Lazeron RHC, Van Huffel S, Thijs RD (2019) Ictal autonomic changes as a tool for seizure detection: a systematic review. Clin Auton Res 29(2):161-181. https://doi.org/10.1007/s10286-018-0568-1

4. Suresh S, Duerstock BS (2020) Automated detection of symptomatic autonomic dysreflexia through multimodal sensing. IEEE J Transl Eng Heal Med 8:1-8. https://doi.org/10.1109/jtehm .2019.2955947

5. Wang X, Ma M, Zhou L et al (2020) Autonomic ganglionic injection of a-synuclein fibrils as a model of pure autonomic failure a-synucleinopathy. Nat Commun 11:1-13 